\title{
Nonlinear waves in hyperbolic metamaterials: focus on solitons and rogues
}

\author{
A. D. Boardman, V. V. Grimalsky, T. Guo, B. Kibler, J. \\ McNiff, et al.
}

A. D. Boardman, V. V. Grimalsky, T. Guo, B. Kibler, J. McNiff, I. Nefedov, Y. Rapoport, C. Argyropoulos, C. Valagiannopoulos, "Nonlinear waves in hyperbolic metamaterials: focus on solitons and rogues," Proc. SPIE 10671, Metamaterials XI, 106710L (7 May 2018); doi: 10.1117/12.2306937

SPIE. Event: SPIE Photonics Europe, 2018, Strasbourg, France 


\title{
Nonlinear waves in hyperbolictedeamaterials: focus on solitons and rogues
}

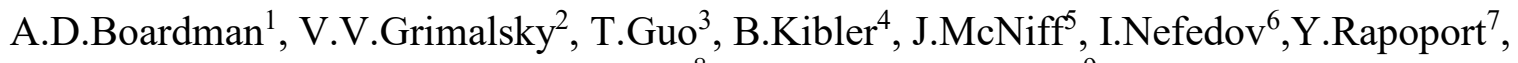 \\ C.Argyropoulos ${ }^{8}$, C.Valagiannopoulos ${ }^{9}$ \\ ${ }^{1}$ Joule Physics Laboratory, University of Salford, Manchester, UK \\ ${ }^{2}$ Autonomous University of State Morelos (UAEM), CIICAp, IICBA, Cuernavaca, 62209, Morelos, \\ Mexico \\ ${ }^{3}$ University of Nebraska-Lincoln (United States) \\ ${ }^{4}$ Laboratoire Interdisciplinaire Carnot de Bourgogne, UMR6303 CNRS/Université Bourgogne \\ Franche- Comté, Dijon, France \\ ${ }^{5}$ Original Perspectives Ltd, Tewkesbury, United Kingdom \\ ${ }^{6}$ Aalto University, School of Electrical Engineering, PO Box 13000, FI-00076 Aalto, Finland \\ ${ }^{7}$ Taras Shevchenko National University of Kyiv, Kyiv, Ukraine \\ ${ }^{8}$ University of Nebraska-Lincoln (United States) \\ ${ }^{9}$ Nazarbayev University, School of Science and Technology, Astana Kasakhstan
}

\begin{abstract}
The investigation of hyperbolic metamaterials, shows that metal layers that are part of graphene structures, and also types I and II layered systems, are readily controlled. Since graphene is a nicely conducting sheet it can be easily managed. The literature only reveals a, limited, systematic, approach to the onset of nonlinearity, especially for the methodology based around the famous nonlinear Schrödinger equation [NLSE]. This presentation reveals nonlinear outcomes involving solitons sustained by the popular, and more straightforward to fabricate, type II hyperbolic metamaterials. The NLSE for type II metatamaterials is developed and nonlinear, non-stationary diffraction and dispersion in such important, and active, planar hyperbolic metamaterials is developed. For rogue waves in metamaterials only a few recent numerical studies exist. The basic model assumes a uniform background to which is added a time-evolving perturbation in order to witness the growth of nonlinear waves out of nowhere. This is discussed here using a new NLSE appropriate to hyperbolic metamaterials that would normally produce temporal solitons. The main conclusion is that new pathways for rogue waves can emerge in the form of Peregrine solitons (and near-Peregrines) within a nonlinear hyperbolic metamaterial, based upon double negative guidelines, and where, potentially, magnetooptic control could be practically exerted.
\end{abstract}

Keywords: nonlinear waves, hyperbolic, solitons, rogues

\section{INTRODUCTION}

Electromagnetic wave propagation in metamaterials is an important area ${ }^{1-6}$. Indeed, it is a vital feature of a global revolution in science. Like many new ideas in science, the suggestion that dramatic artificial materials could be created that could cloak objects and lead to perfect lenses was already known before it was launched properly in 1999, when Rodger Walser', from the University of Texas, published the word 'metamaterial'. This fascinating name for artificial material that exhibits properties deriving from its composition, instead of its fundamental intrinsic physical property, has attracted a worldwide interest. For the visible light domain, it means that the size of the intrinsic components of metamaterials must be much smaller than any excitation wavelength. As a consequence, there is a huge global demand to build nanostructures for the optical domain. The metamaterial family is growing, in general, but the hyperbolic members are popular because they have uniaxial symmetry and an isofrequency surface that is a hyperboloid. Physically, they are relatively easy to construct in layered form, with the optic axis parallel to the z-axis. This means that they could have a permittivity tensor that is diagonal, with positive $x$ and $y$ components and a

Metamaterials XI, edited by Allan D. Boardman, Anatoly V. Zayats, Kevin F. MacDonald, Proc. of SPIE Vol. 10671, 106710L · C 2018 SPIE · CCC code: 0277-786X/18/\$18 · doi: 10.1117/12.2306937 
negative $z$ component. However, there is so much versatility for this type of construction that it helps to identify artificial metamaterials called type I hyperbolics that have just one component of the dielectric tensor that is negative. This means that, in the same, easy to construct, hyperbolic family, there is also a type II that has two negative tensor components.

For this article, after this initial and very broad introduction, section 2 discusses a graphene-based version of a hyperbolic metamaterial, while section 3 bases everything around a dielectric layered system, for which an effective medium approach is then adopted. This leads to a version of the widely known nonlinear Schrödinger equation (NLSE). It will enable a beautiful discussion of what have become known, in popular terminology, as rogue waves. They can be stimulated in straightforward manner by opening up the NLSE using a constant background perturbed by a tiny periodic disturbance. This evolves to what is widely known as a Peregrine soliton ${ }^{6}$. The latter is named after Howell Peregrine, who, back in 1983, at the University of Bristol, looked brilliantly at these waves and found that they appear to come out of nowhere and then disappear quite suddenly again. In hydrodynamics, this has been known for some time with waves leaping suddenly out of what seems to be a calm sea. They are called 'rogue waves' because they jump out from what seems to be a flat ocean. They then keep on rising enough to damage floating objects like ships and then simply disappear. This will be discussed, from a metamaterial point of view, in section 4 . The techniques that need developing for hyperbolic metamaterials will be quite advanced, so the tools involving double negative metamaterials will be deployed, because thy reveal significant pathways towards outcomes for hyperbolic metamaterials. The full rogue wave details of the latter will be investigated in the near future.

\section{NEGATIVE REFRACTION IN LAYERED STRUCTURES WITH HYPERBOLIC DISPERSION}

Negative refraction is an intriguing and counter-intuitive phenomenon that has attracted much attention during the last two decades. Apart from making the light to bend along directions not expected from our everyday experience, it carries a bunch of amazing properties leading to designs surpassing the known limitations of performance such as the perfect planar lens ${ }^{7}$. A prerequisite for negative refraction is to emulate negative permittivity accompanied by negative effective permeability ${ }^{8}$; however, for thin structures compared to the operational wavelength the demand for opposite magnetic response can be relaxed ${ }^{9}$. Therefore, one can achieve high-quality negative refraction in magnetically inert structures such as multilayers with hyperbolic properties.

It is well known that hyperbolic media are called the materials which are characterized by a positive effective permittivity along the one direction and a negative one along the other direction; they are taking their name from the shape of iso-frequency contour levels of their dispersion relation. The most direct way to fabricate a structure emulating hyperbolic media properties is to stack parallel layers or sheets of plasmonic materials or even perfect metals ${ }^{10}$ into an ordinary host of positive permittivity. If that lattice is very densely populated and deeply subwavelength, then effective-medium approximation ${ }^{11}$ is applicable and the permittivity along the normal-tointerfaces direction is found close to that of the background material (and thus positive), while the permittivity parallel to the interfaces becomes negative.
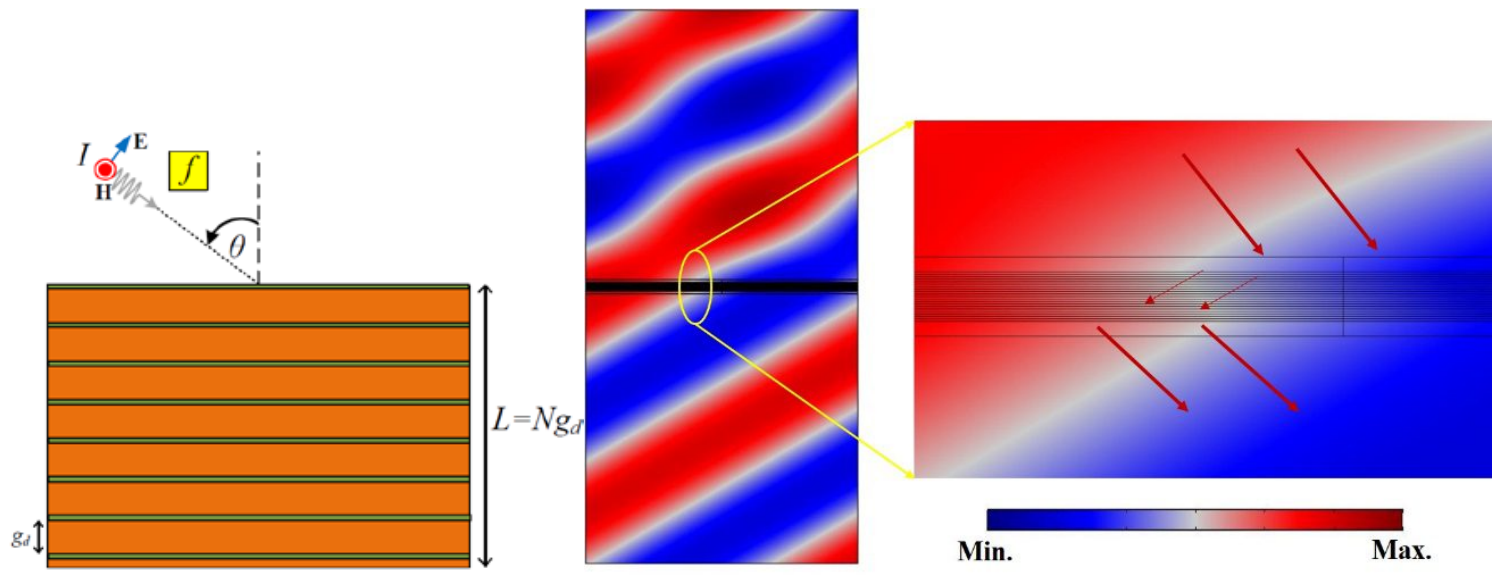

Fig. 2.1: (a) The layered design of graphene multilayers excited by obliquely incident THz light. (b) Demonstration of negative refraction through field spatial distribution. 
The role of plasmonic layers in hyperbolic media can be well-played by graphene ${ }^{12}$ which exhibit such properties at $\mathrm{THz}$ frequencies and can be electrically characterized by its surface conductivity $\sigma\left(f, \mu_{c}\right)$, where $\mu_{c}$ is the chemical potential. Therefore, the structure to be considered in this part is exactly this: $N$ graphene monolayers of surface conductivity $\sigma$ (measured in $1 / \Omega$ ) with distance $g_{d}$ between them which are placed into a dielectric background of relative permittivity $\varepsilon_{d}$, as shown in Fig. 2(a). The thickness of the device is obviously equal to $L=N g_{d}$ and the excitation is an oblique plane wave of intensity $I$ forming an incidence angle $\theta$ with the interfaces. Throughout this part and only if otherwise specified, we always assume the chemical potential of graphene sheets $\mu_{c}=50 \mathrm{meV}$, the dimensions $g_{d}=0.1 \mathrm{um}, \mathrm{N}=20$, and dielectric permittivity $\varepsilon_{d}=2.2$. In addition, both graphene and dielectric are assumed to be lossless in all our calculations. Obviously, the one of the electric field components should be normal to the air/medium boundary so that the dielectric anisotropy gets activated. Time dependence of the form $e^{+2 \pi f t}$ is suppressed.

In order to demonstrate clearly the effect of negative refraction, we compute the field distribution of the layered structure in Fig. 2(b), with incident angle $\theta=30^{\circ}$. Clearly, the oblique illumination gets bended into the layered structure with negligible reflections. The angle of transmission $\theta_{T}$ can be computed with the spatial shift ${ }^{13} \Delta$ $\operatorname{as} \theta_{T}=\tan ^{-1}(\Delta / L)$, where $\mathrm{L}$ is the total length of the

computed with the spatial shift $\Delta \operatorname{as} \theta_{T}=\tan ^{-1}(\Delta / L)$, where ${ }^{14} \mathrm{~L}$ is the total length of the
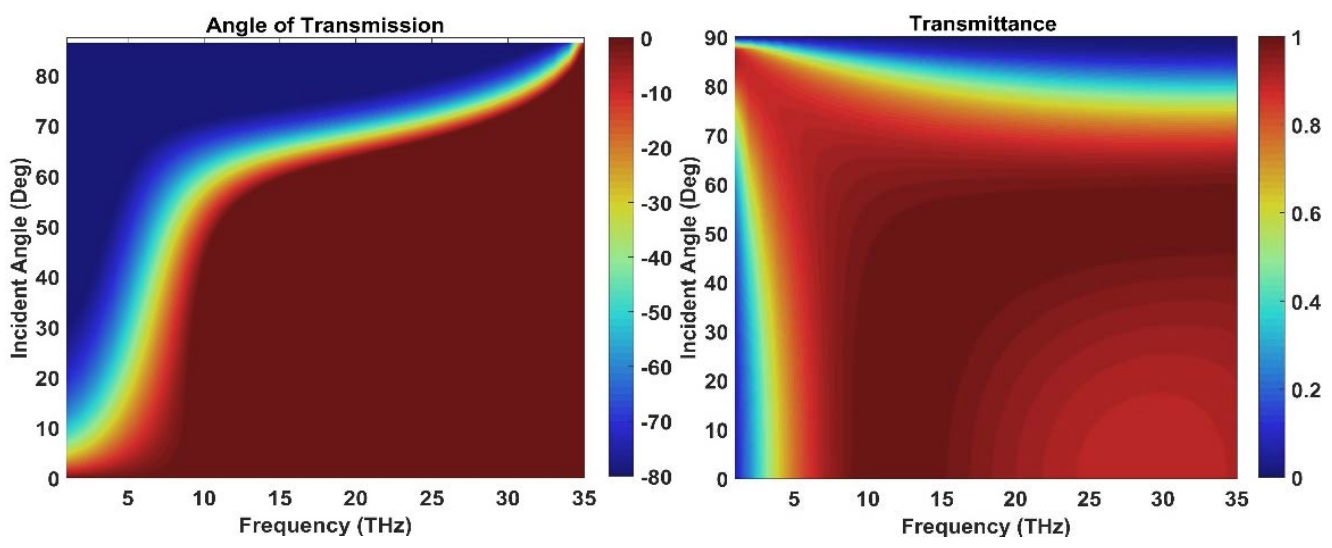

Fig. 2.2: Variations with respect to operating frequency and incidence angle of: (a) the angle of transmission and (b) transmittance. Linear structure.

mutilayered structure. If the transmission angle is smaller than zero, we have negative refraction. In the linear regime (when non-linearities are absent from both the dielectric host and the graphene), the results can be analytically obtained and are represented in Figs. 2.2. 


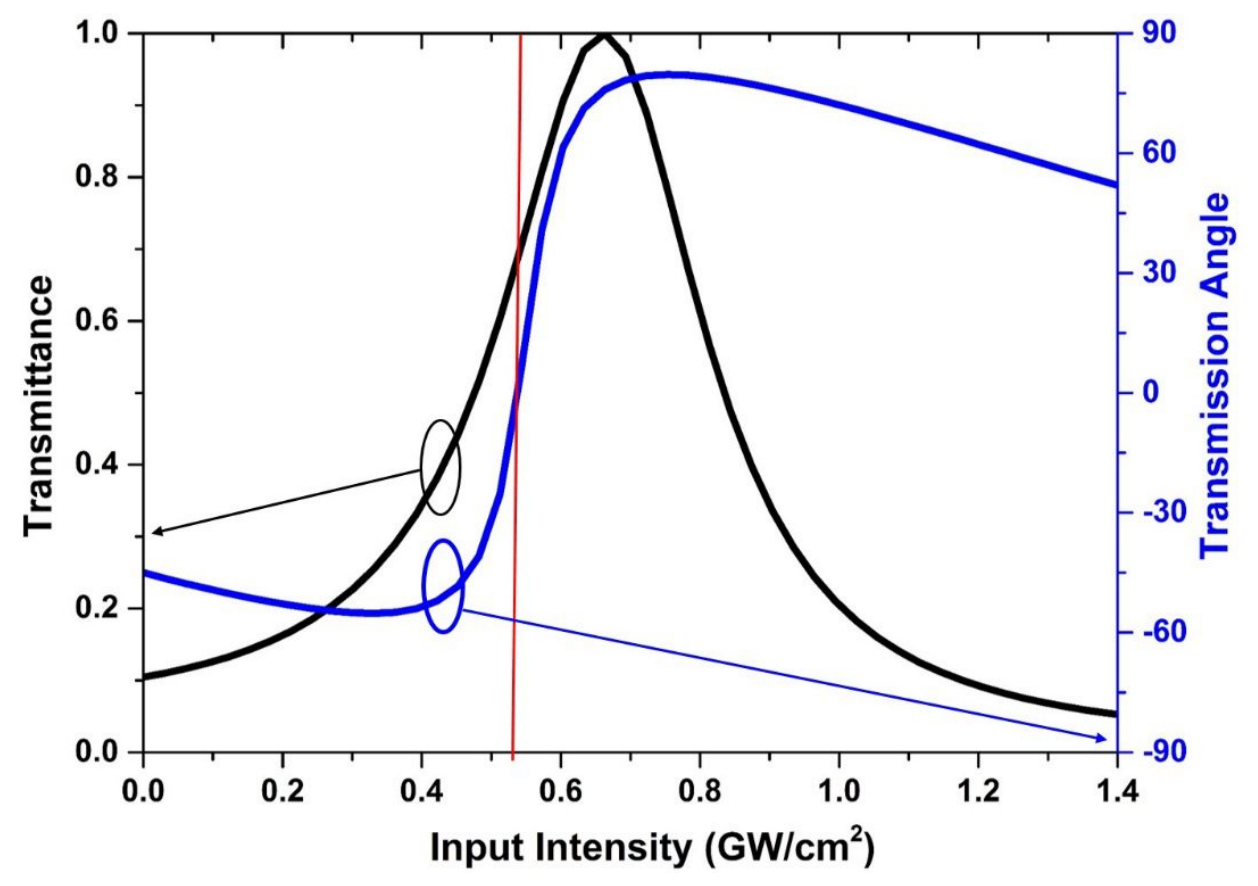

Figure 2.3: The computed transmittance (black line) and transmission angle (blue line) versus the input intensity of the non-linear graphene hyperbolic metamaterial

In particular, in Fig. 2.2(a) we show the transmitting angle $\theta_{T}$ into the graphene multilayers as function of the operational frequency $f(\mathrm{THz})$ and the incidence angle $\theta$. It is remarkable that substantial anomalous refraction is recorded for smaller frequencies and more oblique rays. When $f \cong 5 \mathrm{THz}$, we have $\theta_{T}<0$ for most of the incidence angles, while for $\theta>75^{\circ}$, the phenomenon is present across the entire band of frequencies; however, let us find what portion of power corresponds to this behavior. For this reason, in Fig.2.2(b), we show the variation of the transmitted power on the same $(f, \theta)$ map that is used in Fig. 2.2(a). It can be noticed that for small $f$ and large $\theta$, the transmittance vanishes meaning that a trade-off between negative refraction and high transmission is detected. However, significance transmittance and simultaneously sizeable negative $\theta_{T}$ is occurred for large incidence angles and small oscillating frequencies.

In Fig. 2.3, we show results obtained through simulations of the Kerr third-order non-linear structure, where the nonlinear properties of graphene were considered, for incident illumination at $\theta=60^{\circ}$ and incident frequency at $5 \mathrm{THz}$. The formula of graphene third-order nonlinear conductivity can be found in ${ }^{14}$. Here, the chemical potential of graphene sheet is $0.9 \mathrm{eV}$. By inspecting the blue line in Fig. 2.3, one can clearly understand how input intensity $I$ changes the type of refraction. The vertical red line marks the $\theta_{T}=0$ regime (normal transmittance). More specifically, an abrupt transition from backward to forward propagation is occurred for $I \cong 0.53 \mathrm{GW} / \mathrm{cm}^{2}$, which makes our non-linear multilayers suitable for switching applications. The black line in Fig. 2.3 represents the transmittance as function of input intensity $I$, where a resonance-like behavior is exhibited. We can observe that negative refraction can be combined with non-negligible transmission, while $100 \%$ transmittance is occurred for a refraction angle $\theta_{T} \cong 80^{\circ}>\theta$. It is clear that controllability of wave steering and direction of propagation is possible by altering the input power.

Nonlinearity can be very useful in tuning, adjusting and specifically tailoring negative refraction in simple hyperbolic structures. Accordingly, the appearance of solitons can play a crucial role in the optimal operation of them. Having now set up an approach based upon graphene the investigation of dielectric layers will now be addressed. This pathway offers a relatively straightforward construction opportunity and the next section addresses the basic nonlinear Schrodinger equation that has well-known soliton solutions but hotspots will be discussed as a special outcome. 


\subsection{Introduction}

\subsection{AN APPPROACH TO HYPERBOLIC METAMATERIALS}

In the present section, we consider nonlinear diffraction ${ }^{15,16}$ in active planar hyperbolic metamaterials $\mathbf{s}^{17,18,19}$ that can be extremely anisotropic. If we consider a metamaterial to be constructed artificially from unit cells that are composed of layers then Fig 3.1 shows a typical example. We can see immediately that setting the tensor elements unequal to each other brings in an anisotropic property. Hyperbolic metamaterial is considered here to be a periodical medium made up of elementary cells with each having three layers, as shown in Fig.3.1. The dielectric tensors for each layer are diagonal and the third layer is active to combat loss. One approach to analyzing this is a metamaterial method by averaging over the unit cells. A more accurate approach is to solve the problem precisely without averaging. It is interesting that these two approaches are actually complimentary and not contradictive to each other. Also making the metamaterial nonlinear leads to valuable conclusions. As always, loss can be a factor that impedes strong conclusions so, here, without loss of generality, we will consider only the case of exact mutual compensation. In the moderately nonlinear regime, a possibility of the formation of hot spots is also investigated.

\subsection{Basic Equations}

We consider now the nonlinear propagation of electromagnetic waves in the kind of layered medium shown.in

Fig.3.1. The system has an overall thickness $\mathrm{L}_{z}$, and a unit cell consisting of 3 alternating elementary layers of

thicknesses $d_{1}, d_{2}, d_{3}$. This is shown clearly in Fig. 3.1.

Note that the anisotropy of the metamaterial being created arises from the multilayer structure as opposed to the properties of the media used in the individual layers.

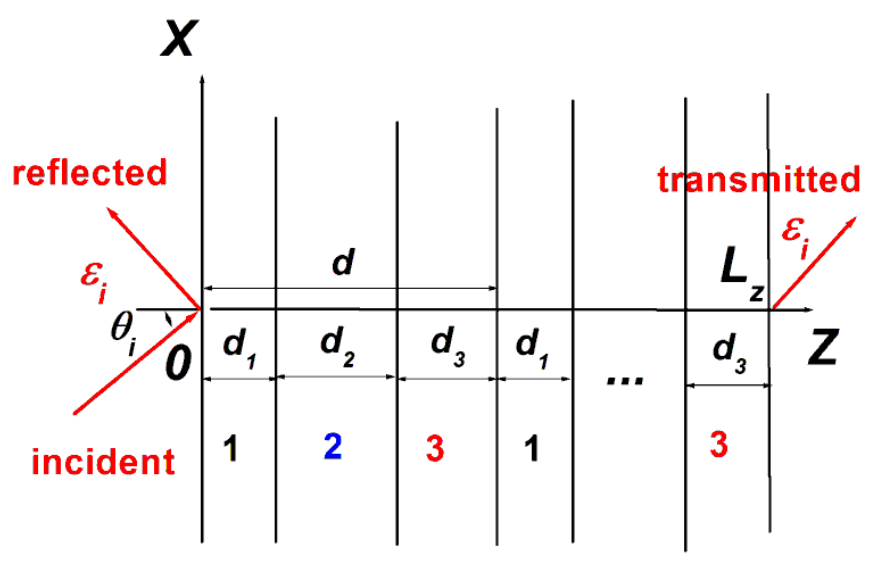

Fig 3.1. Geometry of the problem. The elementary cell includes 3 layers. Layer 1 possesses $\varepsilon_{L I}<0$, layers 2 , 3 possess $\varepsilon_{L 2,3}>0$.

The thickness of the unit cell is $d=d_{1}+d_{2}+d_{3}$ and, along $O Y$ axis, the system is completely uniform. The propagating nonlinear modulated waves have components $E_{x}, H_{y}, E_{z}$ and they all vary with an angular frequency $\omega>0$, with the form $\sim \exp (-i \omega t)$, where $t$ is time. Within each layer, the dependence of the permittivity of the electric field is ${ }^{20}$ 


$$
\begin{aligned}
& \varepsilon(z, x)=\varepsilon_{L}(z)+\frac{\alpha(z)|E|^{2}}{1+\gamma(z)|E|^{2}} \\
& |E|^{2} \equiv\left|E_{x}\right|^{2}+\left|E_{z}\right|^{2}
\end{aligned}
$$

A model of local saturating nonlinearity is used here. Each elementary layer is assumed to be uniform, so $\varepsilon_{L}, \alpha, \gamma$ are step-like functions of $z$. Generally, $\varepsilon_{L}$ and $\alpha$ are complex, because possible dissipative and active media are going to be investigated. The saturation coefficient of the of the nonlinearity is assumed to be real and positive, so that $\gamma>0$.

The Maxwell equations assumed here involving, respectively, the time t, magnetic field vector $\vec{H}$, the displacement vector $\vec{D}$, the electric field vector $\vec{E}$ and the velocity of light, $c$, for a vacuum, are

$$
\nabla \times \vec{H}=\frac{1}{c} \frac{\partial \vec{D}}{\partial t} ; \quad \nabla \times \vec{E}=-\frac{1}{c} \frac{\partial \vec{H}}{\partial t}
$$

All lengths will be normalized to $l_{n}=1 \mu \mathrm{m}$, and time is normalized to $t_{n}=l_{n} / c$. The connections between the components of the electromagnetic field are, together with the equation for the slowly varying magnetic field amplitude

$$
\begin{aligned}
& E_{x}=-\frac{i \beta}{\omega} \frac{\partial H}{\partial z} e^{-i \omega t}, H_{y}=H e^{-i \omega t}, E_{z}=\frac{i \beta}{\omega} \frac{\partial H}{\partial x} e^{-i \omega t} ; \beta(z, x) \equiv \frac{1}{\varepsilon(z, x)} \\
& \frac{\partial H}{\partial t}-\frac{i}{2 \omega} \frac{\partial}{\partial x}\left(\beta \frac{\partial H}{\partial x}\right)-\frac{i}{2 \omega} \frac{\partial}{\partial z}\left(\beta \frac{\partial H}{\partial z}\right)-\frac{i \omega}{2} H=0
\end{aligned}
$$

An approach will now be applied where the averaged values of the permittivity are introduced and, therefore, takes the view that the hyperbolic metamaterial is an effectively continuous medium. Accurately speaking, this approach is applicable, when the typical width of the layers is much less than the wavelength in the medium. For this model, it is assumed that the elementary layers 2, 3, are nonlinear, whereas the first one is set to be linear. The non-dimensional units can be used, with $c=1$, so that for this averaged medium we have, at each point of it,

$$
E_{x}=-\frac{i \beta_{x}}{\omega} \frac{\partial H}{\partial z} ; \quad E_{z}=\frac{i \beta_{z}}{\omega} \frac{\partial H}{\partial x}
$$

$E_{x}, E_{z}$ are now the average values of the components of the electric field i.e. $E_{x} \equiv\left\langle E_{x}\right\rangle, E_{z} \equiv\left\langle E_{z}\right\rangle$. Hence, the equation for the slowly varying amplitude $H(z, x, t)$ is now

$$
\frac{\partial H}{\partial t}-\frac{i}{2 \omega} \frac{\partial}{\partial x}\left(\beta_{z} \frac{\partial H}{\partial x}\right)-\frac{i}{2 \omega} \frac{\partial}{\partial z}\left(\beta_{x} \frac{\partial H}{\partial z}\right)-\frac{i \omega}{2} H=0
$$

Actually, the amplitude $H(z, x, t)$ is slowly varying with respect to time $t$ only. The formulae for the averaged components of the inverse permittivity are: 


$$
\begin{aligned}
& \beta_{z}=\beta_{1} \frac{d_{1}}{d}+\beta_{2} \frac{d_{2}}{d}+\beta_{3} \frac{d_{3}}{d}, \quad \beta_{1,2,3}=\frac{1}{\varepsilon_{1,2,3}} ; \\
& \varepsilon_{x}=\varepsilon_{1} \frac{d_{1}}{d}+\varepsilon_{2} \frac{d_{2}}{d}+\varepsilon_{3} \frac{d_{3}}{d}, \quad \beta_{x}=\frac{1}{\varepsilon_{x}} .
\end{aligned}
$$

These formulae are applied at each point of the averaged medium, where the values of $E_{x}, E_{z}$ are computed. $d_{1,2,3}$ are the thicknesses of the elementary layers and $d=d_{1}+d_{2}+d_{3}$. Only the ratios $d_{1,2,3} / d$ are important and not their absolute values. In addition, it is supposed that the change of the field components $E_{x, z}, H_{y}$, as well as of the values $\beta_{x, z}$ along the layers (in the direction $x$ ) happen over lengths, much larger than the thicknesses of the layers $d_{1,2,3}$. Within this approximation, the nonlinear permittivities of the elementary layers 2,3 become:

$$
\begin{aligned}
& \varepsilon_{2}=\varepsilon_{2 L}+\frac{\alpha_{2} \cdot\left(\left|E_{2 x}\right|^{2}+\left|E_{2 z}\right|^{2}\right)}{1+\gamma_{2} \cdot\left(\left|E_{2 x}\right|^{2}+\left|E_{2 z}\right|^{2}\right)} \\
& \varepsilon_{3}=\varepsilon_{3 L}+\frac{\alpha_{3} \cdot\left(\left|E_{3 x}\right|^{2}+\left|E_{3 z}\right|^{2}\right)}{1+\gamma_{3} \cdot\left(\left|E_{3 x}\right|^{2}+\left|E_{3 z}\right|^{2}\right)}
\end{aligned}
$$

in which $E_{2 x}$ etc. are the components of the electric field in the corresponding elementary layers near the specific point within the averaged medium. There is the problem of the correspondence between the averaged values $E_{x}$, $E_{z}$ and $E_{2 x}, E_{3 x}, E_{2 z}, E_{3 z}$ within the elementary layers. However, the tangential component of the electric field are continuous in the layered medium

$$
E_{2 x}=E_{3 x}=E_{x}
$$

Also, the normal component of the electric induction is also continuous so that

$$
D_{2 z}=D_{3 z}=<D_{z}>\equiv \frac{E_{z}}{\beta_{z}}
$$

Consequently,

$$
E_{2 z}=\beta_{2} D_{2 z}=\frac{\beta_{2}}{\beta_{z}} E_{z} ; E_{3 z}=\beta_{3} D_{3 z}=\frac{\beta_{3}}{\beta_{z}} E_{z}
$$

This process describes the whole metamaterial as a continuous, effective, medium. Therefore, the boundary conditions between the elementary layers included in the metamaterial are not applied, but the boundary conditions at the boundaries $\mathrm{z}=0$ and $\mathrm{z}=L_{z}$ between the layer of the metamaterial and surrounding medium are still necessary, and are applied.

The metamaterial, consists of periodically alternation isotropic layers. However, the anisotropy, which is evident, has a clear physical sense. First of all, for a linear medium, the formulae have been derived accurately, using the consideration of the periodical media with the further approximation of thin layers. Qualitatively, the description of the anisotropy of the hyperbolic metamaterial may be drawn from the equivalent transmission line, describing a planar multilayered metamaterial ${ }^{21}$. In accordance with this approach, averaged transverse field 
components ( $E_{x}, H_{y}$ in this case ) would be determined through an average value of the effective impedance, which is proportional to $\left\langle\varepsilon_{x x}\right\rangle$, where the brackets $\langle\ldots\rangle$ mean a proper averaging (in our case over the period of the structure) of the value placed inside the brackets. On the other hand, using an averaged longitudinal field component, in this case $E_{z}$, it would be determined through the value proportional to $\left\langle\varepsilon_{z z}^{-1}\right\rangle$. For the hyperbolic metamaterial, based on the isotropic alternating layers, in each, $\varepsilon_{x x}=\varepsilon_{z z}$, while the signs of $\varepsilon_{x x}$ alternate from layer to layer belonging to each cell of the structure. Providing that $\left\langle\varepsilon_{x x}\right\rangle>0$, it is easy to see that $\left\langle\varepsilon_{x x}^{-1}\right\rangle=\left\langle\varepsilon_{z z}^{-1}\right\rangle<0$. Therefore, we obtain a hyperbolic uniaxial metamaterial with opposite signs of the diagonal tensors of the dielectric permittivity. In the nonlinear case, the periodicity of the medium, which is the basis of an averaging, disappears. Nevertheless, the relations with the same form as that for the linear case, can be used phenomenologically, accounting for a contribution of the nonlinearity into the values $\varepsilon_{2,3}$ and, respectively into $\beta_{1,2,3}, \varepsilon_{x, z}$, as it is described above.

The exact equations have been solved by the finite differences method, where the operator factorization, or the method of Douglas - Rachford ${ }^{22}$ has been applied. For this case, the slowly varying amplitude equation can be written symbolically as

$$
\begin{aligned}
& \frac{\partial H}{\partial t}+\hat{L}_{1} H+\hat{L}_{2} H=0, \quad \text { where } \hat{L}_{1} H \equiv-\frac{i}{2 \omega} \frac{\partial}{\partial z}\left(\beta \frac{\partial H}{\partial z}\right)-\frac{i \omega}{2} H \\
& \hat{L}_{2} H \equiv-\frac{i}{2 \omega} \frac{\partial}{\partial x}\left(\beta \frac{\partial H}{\partial x}\right)
\end{aligned}
$$

The following notation is used:

$$
\chi^{p+1} \equiv \frac{H^{p+1}-H^{p}}{\tau}
$$

Here $\tau$ is the step for time $t, t^{p} \equiv p \cdot \tau, p=0,1,2 \ldots ; H^{p} \equiv H\left(t^{p}\right)$. The above equations can be represented as:

$$
\begin{aligned}
& \left(1+\tau \hat{L}_{2}\right) \chi^{p+1 / 2}=-\left(\hat{L}_{1}+\hat{L}_{2}\right) H^{p} \\
& \left(1+\tau \hat{L}_{1}\right) \chi^{p+1}=\chi^{p+1 / 2} \\
& H^{p+1}=H^{p}+\tau \chi^{p+1}
\end{aligned}
$$

The boundary conditions are also rewritten in terms of $\chi^{p+1}$. The operators $\hat{L}_{1,2}$ are approximated by finite differences. The inverse permittivity $\beta$ is a piecewise-continuous function of $z$, so the values of $\beta$ are approximated between the nodes for $H$ : if the values of $H$ in the nodes are $H_{j} \equiv H\left(z_{j}\right) \equiv H\left(j \cdot h_{z}\right), j=0,1,2 \ldots$, then $\beta_{j+1 / 2} \equiv$ $\beta\left((j+1 / 2) \cdot h_{z}\right)$. Each distance $d_{1,2,3}$ includes integral numbers of spatial steps $h_{z}$. To take into account the nonlinearity, the iterations have been applied. This method is unconditionally stable.

The system is limited in $\mathrm{x}$-direction, $0 \leq x \leq L_{x}$, so the boundary conditions at $x=0$ and $x=L_{x}$ are $H=0$.

The boundary conditions have been approximated by finite differences too. They are applied at the second fractional step in , to compute $\chi^{p+1}$. The derivatives $\partial \chi^{p+1} / \partial x$ are calculated in the positive direction of $x$, because $v_{x}>0$. In the point $x_{l} \equiv l \cdot h_{x}$ the approximation is $\partial \chi^{p+1} / \partial x \approx\left(\chi^{p+1}{ }_{l}-\chi^{p+l}{ }_{l-1}\right) / h_{x}$. This approximation makes it possible to calculate $\chi^{p+l}$ from smaller values of $x$ to higher ones: $l=1,2,3, \ldots$, and to use for computing $\chi^{p+1}$ the value of $\chi^{p+1}{ }_{l-1}$, which has been just calculated.

The implicit-explicit methods, like the Peaceman - Rachford one ${ }^{22}$, do not provide good stability in our nonlinear case, as our simulations demonstrated. The schemes like splitting with respect to physical factors, or the summitry approximation, require small temporal steps and therefore are practically unusable here. 
The incident wave is assumed as a beam bounded in $x$-direction:

$$
A_{i}(z=0, x, t)=A_{0} \exp \left(-\left(\frac{x-x_{1}}{x_{0}}\right)^{2}\right) \tanh \left(\frac{t}{t_{0}}\right)
$$

The temporal dependence is tanh-like and the maximum amplitude of the incident wave tends to $A_{0}$ at the boundary $z=0$. Below, the established values of the electromagnetic field are presented. As it will be shown , strongly nonlinear phenomena, namely hot spot formation is possible for the present system with the corresponding parameters. This is the case when a possibility of stationary solution is not evident beforehand, and to prove such a possibility, the method of establishing (steady-state solution) and, respectively, the initialboundary condition are quite adequate.

\subsection{Details of the structure}

The simulations have been done for hyperbolic media. For linear EM waves the parameters of the elementary layers are chosen to get $\varepsilon_{x}{ }^{\prime}>0, \varepsilon_{z}{ }^{\prime}<0$, where $\varepsilon^{\prime} \equiv \operatorname{Re}(\varepsilon)$. The medium 1 is with $\varepsilon_{L I}{ }^{\prime}<0,1,2$ and 3 are with $\varepsilon_{L I}$ ' $>0$. The hyperbolic media possess the properties, which are important both for theoretical and practical views. Below it is assumed that the real parts of the media 2,3 are equal: $\varepsilon_{L 2}{ }^{\prime}=\varepsilon_{L 3}$ '.

\subsubsection{Requirements for materials}

The materials for the elementary layers satisfy the following requirements: $\mid \varepsilon_{L 1}$ ' $<\varepsilon_{L 2}$; the dissipation within each layer is made as small possible.

In the near-infrared and visible optical range (wavelengths $\lambda_{0}=0.5-2 \mu \mathrm{m}, \omega=5 \cdot 10^{14}-4 \cdot 10^{15} \mathrm{~s}^{-1}$ ), the medium 1 can be metallic with high conductivity, or semi-metallic, like $\mathrm{Ag}, \mathrm{Au}, \mathrm{Cu}, \mathrm{Bi}$. The linear effective permittivity is:

$$
\varepsilon_{l 1}=\varepsilon_{\text {lattice }}-\frac{\omega_{p}{ }^{2}}{\omega(\omega+i v)} ; \quad \omega_{p}^{2} \equiv \frac{4 \pi e^{2} n_{0}}{m^{*}}
$$

where $\omega_{p}$ is the plasma frequency, $n_{0}$ is the electron concentration, $m *$ is the effective electron mass, $v$ is the electron collision frequency.

There are critical parameters, such as the electron concentration $n_{0}$. This should be preferably $n_{0}=10^{21}-$ $2 \cdot 10^{22} \mathrm{~cm}^{-3}$, to provide the negative effective permittivity in the corresponding layer(s). Also as required for the hyperbolic metamaterial, in the optical range of moderate absolute values, $\varepsilon_{L I}$ ' $=-3--10$. Hence, the effective permittivity is negative due to the electron plasma. Thus $0.2 \omega_{p}<\omega<0.5 \omega_{p}$. The collision frequency should be relatively small i.e. $v \leq 10^{13} \mathrm{~s}^{-1}$.

The layers 2, 3 should be dielectrics with high (with a positive real part) permittivity in the optical range and low losses, or even active to provide amplification). Examples are $\mathrm{Hf}_{2} \mathrm{O}_{5}, \mathrm{Ta}_{2} \mathrm{O}_{5}, \mathrm{Al}_{2} \mathrm{O}_{3}, \varepsilon_{L 2}{ }^{\prime} \geq 10$.

In the THz range (wavelengths $\lambda_{0}=0.5-0.03 \mathrm{~mm}$, or $\omega=10^{12}-6 \cdot 10^{13} \mathrm{~s}^{-1}$ ) the narrow-gap semiconductors $n$-InSb, $n$-InAs, $n$ - $\mathrm{Cd}_{1-\mathrm{x}} \mathrm{Hg}_{\mathrm{x}} \mathrm{Te}$ are perspective. The frequency of EM wave should satisfy the inequality $\omega<\mathrm{E}_{g} / \hbar$, where $\mathrm{E}_{g}=0.1-0.4 \mathrm{eV}$ is the forbidden gap for narrow-gap semiconductors. These semiconductors possess small effective electron masses $m^{*}=(0.002-0.02) m_{e}$ and low collision frequencies $v=$ $\left(10^{11}-310^{11}\right) \mathrm{s}^{-1}$ at moderate doping levels $n_{0}=\left(10^{15}-10^{16}\right) \mathrm{cm}^{-3}$. For the layers 2,3 , dielectrics like $\mathrm{TiO}_{2}$, $\mathrm{MoO}_{2}, \mathrm{SrTiO}_{3}, \mathrm{LiNbO}_{3}$ with high permittivities $\varepsilon_{L 2}{ }^{\prime} \geq 20$ can be used. 
The surrounding media at $z<0$ and $z>L_{z}$ are made to possess the permittivity $\mathcal{E}_{i}$ matched to the hyperbolic medium in the linear case. Namely, the value of $\varepsilon_{i}$ has been chosen to provide zero reflection coefficient at $z=0$ in the case of the incidence of the plane wave at the incidence angle $\theta_{i}$. In the experimental realization of a hyperbolic medium, the elementary layer 3 should be active and the results of simulations below are tolerant to changes of the lengths and the widths of the such a medium.

\subsection{Some vital aspects of the simulations.}

In the nonlinear case, the layers 2,3 are assumed to be nonlinear, whereas 1 is linear. One case is considered i.e. $\alpha>0$ shown in Fig.3.2. Here the input beam is subject to essential nonlinear diffraction. Within the hyperbolic media strongly nonlinear waves can occur, namely hotspots are formed for $\alpha<0$ (near $x \approx 13 \mu m, z \approx 27 \mu m$, for example), where the electromagnetic energy concentrates.

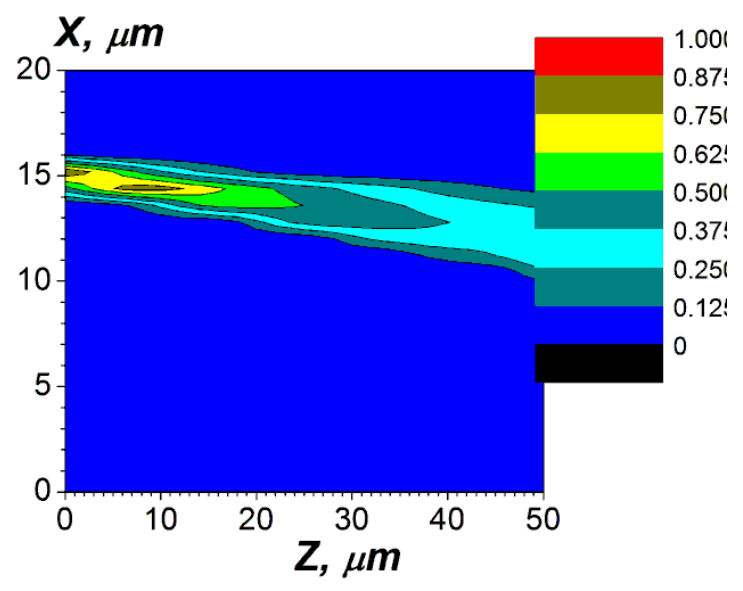

(a)

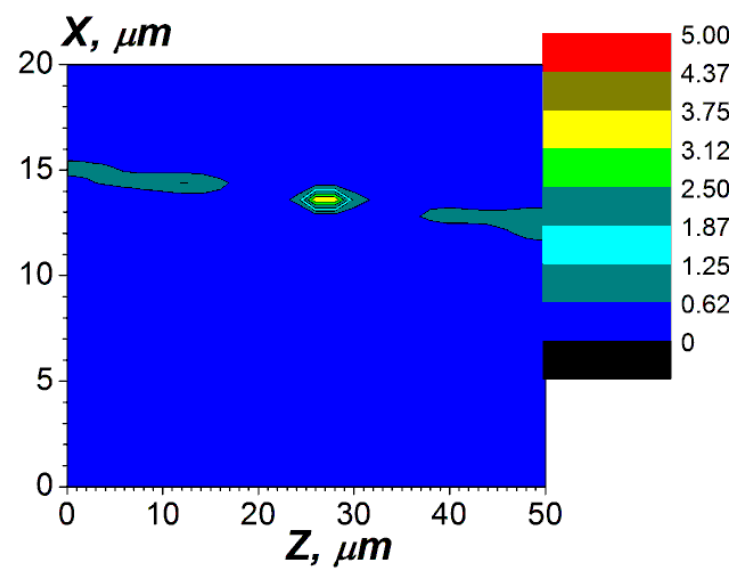

(b)

Fig 3.2 Propagation of nonlinear waves. The circular frequency is $\omega=6 \pi \cdot 10^{14} \mathrm{~s}^{-1}\left(\lambda_{0}=1 \mu \mathrm{m}\right)$.

(a) The nonlinear coefficient for elementary layers 2,3 is $\alpha=1, \gamma=0.5$. Within framework of averaged permittivities and using thicknesses of elementary layers equal to $d_{1}=7.5 \mathrm{~nm}, d_{2}=d_{3}=3.75 \mathrm{~nm}$. The permittivity of the contacting medium is $\varepsilon_{I}=4.203$. The half-width of the incident beam is $x_{0}=1 \mu \mathrm{m}$.

(b) $\alpha=-1$. 
It can be seen that a hotspot is formed with the proper sign of the nonlinearity, namely negative, opposite to Fig.3.2a. From the latter it is clear that solitons are possible so, in the next section, without simulations, a specific algebraic approach that leads to the fundamental equation for them will be discussed. For now, however, we present a hot spot in Fig. $3.2 \mathrm{~b}$ as a simulation example of the stationary regime.

\subsection{The nonlinear Schrodinger equation [NLSE]}

In the case of the moderate nonlinearity, the NSE has the structure

$$
K_{z} \frac{\partial U}{\partial \widetilde{z}}+K_{x} \frac{\partial U}{\partial \widetilde{x}}+i G \frac{\partial^{2} U}{\partial \widetilde{x}^{2}}+i N F\left(|U|^{2}\right) U=0
$$

in which the wave amplitude $U(\widetilde{z}, \widetilde{x})$ is slowly varying, with respect to $\widetilde{z}$. The coefficients $K_{x, z}, N$, and the function $F$ can be expressed through the parameters of the hyperbolic medium and the frequency. Eq. (3.16) has been expressed in the rotated coordinate frame $\widetilde{X} O \widetilde{Z}$, where $O \widetilde{Z}$ axis is aligned along the group velocity direction within the hyperbolic medium. The group velocity $\boldsymbol{V}_{\boldsymbol{g}}$ is obtained from the linear dispersion equation

$$
\begin{aligned}
& D\left(k_{z}, k_{x}, \omega\right)=k_{z}^{2} \beta_{x}+k_{x}^{2} \beta_{z}-\omega^{2}=0, \\
& V_{g x} \equiv-\left(\frac{\partial D}{\partial k_{x}}\right) \cdot\left(\frac{\partial D}{\partial \omega}\right)^{-1}=\frac{k_{x} \beta_{z}}{\omega}<0, \quad V_{g z} \equiv-\left(\frac{\partial D}{\partial k_{z}}\right) \cdot\left(\frac{\partial D}{\partial \omega}\right)^{-1}=\frac{k_{z} \beta_{x}}{\omega}>0, \\
& k_{x}, k_{z}, \omega, \beta_{x}>0, \quad \beta_{z}<0 .
\end{aligned}
$$

Here the frequency dispersion of the components of the inverse permittivity $\beta_{x, z}$ is neglected. However, when the frequency dispersion is taken into account, the signs of the components of the group velocity actually preserve. Hence, for small thicknesses of the elementary layers, in the case of the moderate nonlinearity, the dynamics of the nonlinear beams in the hyperbolic medium can be described by NLSE, where one-directional propagation of an electromagnetic wave is considered.

In the case of the propagation along $O Z$, which is perpendicular to the boundaries of the layers, the structure of the stationary NLSE, for moderate nonlinearity, becomes the standard form

$$
\begin{aligned}
& \frac{\partial D}{\partial k_{z}} \frac{\partial U}{\partial z}-i \frac{\partial D}{\partial\left(k_{x}^{2}\right)} \frac{\partial^{2} U}{\partial x^{2}}-i \frac{d_{1}+d_{2}}{d} \omega^{2} \alpha|U|^{2} U=0 \\
& D\left(k_{z}, k_{x}, \omega\right)=k_{z}^{2} \beta_{x}+k_{x}^{2} \beta_{z}-\omega^{2}=0 \\
& \frac{\partial D}{\partial k_{z}}=2 k_{z} \beta_{x}>0, \quad \frac{\partial D}{\partial\left(k_{x}{ }^{2}\right)}=\beta_{z}<0
\end{aligned}
$$

for the case of moderate nonlinearity with $D=0$ being the linear dispersion equation for electromagnetic waves in a hyperbolic medium. In an equivalent manner, this equation can be written in the form

$$
\frac{\partial U}{\partial z}-i \frac{\beta_{z}}{2 k_{z} \beta_{x}} \frac{\partial^{2} U}{\partial x^{2}}-i \frac{d_{1}+d_{2}}{d} \frac{\omega^{2} \alpha}{2 k_{z} \beta_{x}}|U|^{2} U=0 .
$$

In the case of the negative cubic nonlinearity $\alpha<0$ and the signs of the diffraction and nonlinear coefficients coincide. It is interesting that then bright spatial solitons can be formed. 


\subsection{Conclusions}

The metamaterial averaging approach can be applied when the thicknesses of elementary layers are small and the nonlinearity is moderate. If $d_{i} \leq 0.1 \lambda_{i}$, where $d_{i}$ is the thickness of each elementary layer, and $\lambda_{i} \equiv \lambda_{0} \cdot \mathcal{E}_{i}^{-1 / 2}$ is the wavelength of the electromagnetic wave in this medium, the results of exact simulations and the averaging approach for the hyperbolic medium are the same. This is very useful since it is now possible to reduce the nonlinear equation for electromagnetic wave propagation to the well-known nonlinear Schrödinger equation. At larger thicknesses of elementary layers the differences between the exact solutions and the metamaterial averaging approach is such that the averaging approach is not valid. This is why it is necessary and important to verify the metamaterial approximation for active nonlinear media. Such problems will be a subject of an upcoming paper.

For solving nonlinear problems various difference schemes have been applied. The implicit-explicit method of Peaceman - Rachford does not possess good stability. The method of the summatory approximation needs very small temporal steps and is practically not applicable. It is very interesting, that the method of the operator factorization, or the method of Douglas - Rachford (known more in the hydrodynamics, than in the nonlinear optics), seems the most appropriate.

A future mechanism of compensation of dissipation can be the creation of active dielectric layers by means of inserting quantum dots ${ }^{17}$ with the inversion of energetic levels. The dynamics of nonlinear waves under moderate net amplification is of great interest, very non-trivial. In accordance with these preliminary evaluations, it is going to be the subject of future work. A possibility of hot spot formation is shown. Note that similarly to bullets (spatio-temporal solitons) in ferrite films and metamaterials ${ }^{23}$, hot spots can be considered as a result of collapsed stabilization.

\section{OPTICAL ROGUE WAVES}

Optical rogue waves have been written about, very vigorously, in the literature, both historically, and currently. It emerges that it is the dispersive type of the standard nonlinear Schrodinger equation [NLSE] that has been used with a special boundary condition. Using the name rogue appeals in broader science because it implies that it is something that does not follow a generally accepted path. Hence, in the context of what is being discussed here, in an analogous fashion to the practice in hydrodynamics, we consider optical phenomena that do not follow the traditionally accepted path. In fact, we become concerned with peaks of intensity that appear to spring out from an almost flat background (albeit with a small perturbation) only to decay away again with equal rapidity. One scenario that can be considered is the famous case of a single peak appearing and then disappearing. This is known as a Peregrine soliton.

Contrary to natural expectation, rogues have actually been observed but are comparatively rare events. This is because there is a relatively low probability that any natural physical system can acquire the correct boundary condition that can create a rogue. Indeed, in the field of hydrodynamics, it was, until relatively recently, difficult to make any appropriate measurements at sea. This lack of evidence led, for a long time, to a disbelief about rogue wave events. However, the other side of the coin is that it is possible to generate them in the laboratory. The key to this is that wave dynamics in weakly nonlinear dispersive media, such as optical Kerr media, or on the surface of deep water, can be described by the NLSE. The latter admits general breather solutions on a finite background, i.e. pulsating envelopes that well-mimic the dynamics of rogue waves that may appear from nowhere and disappear without leaving a trace. Metamaterials offer the potential to control a range of electromagnetic behavior in ways that cannot be achieved with materials found in Nature and the ability to create purpose-built materials to fulfil new and novel functionality where these artificial structures, that exert influence over electromagnetic waves at the sub-wavelength level, are extensively being studied and designed. Here we contribute to this development.

The study of rogue waves in metamaterials is currently at an early stage ${ }^{6}$, however. Only a few recent numerical studies, based ona transmission line model (with NLSE reduction) or a cubic- quintic NLSE can be found. In particular, it was shown there is the possibility of producing extreme waveform events, with strong similarities to NLSE breather waves (not only Peregrine ${ }^{6}$, but also Akhmediev, or Kuznetsov-Ma ,breathers). In the following, we investigate the propagation behavior of wave 
excitation corresponding to rogue breather solutions in transparent hyperbolic metamaterials with the inclusion of higher-order dispersive, or nonlinear, effects. Hence the fascinating idea of rogue wave emergence in the form of Peregrine ${ }^{6}$ solitons (and near-Peregrines) within a nonlinear metamaterial environment and where potentially other control could be exerted is developed as a new pathway created by hyperbolic metamaterials.

Both double negative and hyperbolic metamaterials have very interesting properties, the full extent of which has not yet been explored. The more recently developed hyperbolic media offer the prospect of a number of properties that makes them an attractive proposition. This includes the simplified construction of hyperbolic metamaterials over double negative particularly at the nanoscale. In addition they have broader band responses which can be important in areas such as information transfer rates or reducing the number of devices needed to operate across wide frequency bands. They can also, for example, be shaped into very sub-wavelength cavities ${ }^{24,25}$. On the other hand the resonant nature of the double negative media makes them particularly useful in some applications such as sensors ${ }^{26,27}$, where a small shift in the resonant response can readily be detected. Here however the interest is particularly in extreme events and how they might be assessed in both double negative and hyperbolic media.

Given the right conditions rogue wave, or extreme events, are produced from an often innocuous initiating modulation, or starting condition, which as it progresses then develops and changes to produce extreme events. An ideal route for studying these events in both forms of metamaterial requires a numerical tool that does not have to be significantly re-written when switching from one form of metamaterial to the next. It would also allow for the propagation from a range of different starting conditions in the different media. This is the technique that has been adopted here and has already shown great promise. In this approach the metamaterials properties of a media are introduced to the NLSE through the various coefficients such as the self-steepening coefficient. Initially, this has been carried out for double negative metamaterials that also reveals the pathways for hyperbolic media. The general form of the NLSE is similar for both the double negative and hyperbolic cases, so, even though there are important quantitative differences in the associated coefficients, the rogue behavior brought about by the differently structured media will reveal common pathways.

The main approach here is based on a modified extension ${ }^{28}$ of the NLSE that takes into account the metamaterial properties with self-steepening and magnetooptic effects. For double negative media it assumes that the nonlinear behavior discussed originates from an isotropic Kerr dielectric in a weakly guiding planar waveguide structure with a metamaterial core. In contrast, hyperbolic media are uniaxial in nature, which results in different coefficients. The media can again be planar, however, and propagation can be orthogonal to the (sub-wavelength) layers, resulting in an averaging of the properties of the individual layers in the electromagnetic response. Fig 4.1(a) shows a typical guiding structure for a double negative nonlinear metamaterial. Fig 4.1(b) shows the uniaxial hyperbolic metamaterial. It is clear, however, that future directions for rogue work can be estimated from an analysis of a double negative metamaterial model. 


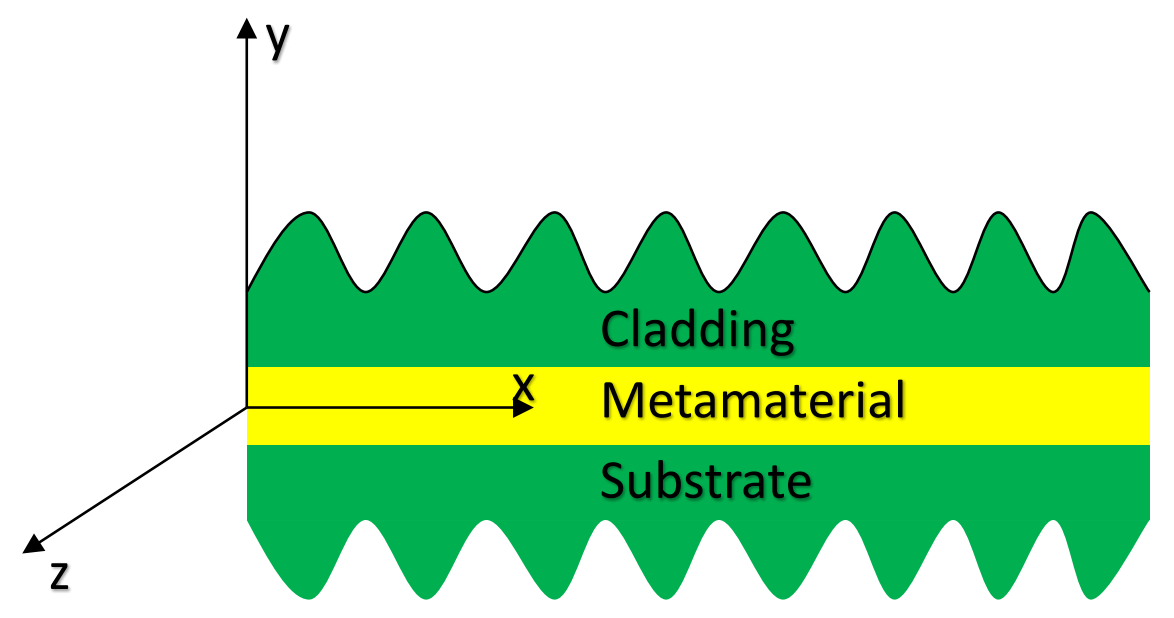

(a)

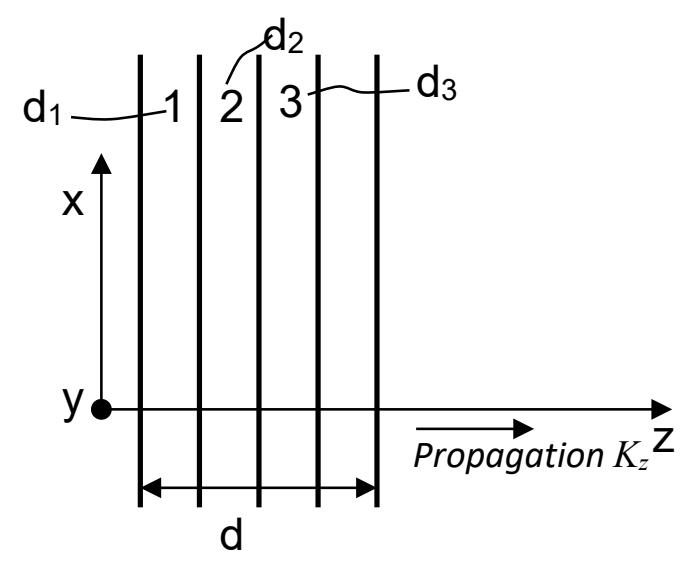

(b)

Figure 4.1. (a) Typical guiding structures for a double negative media with the nonlinear metamaterial in the central region and (b) Hyperbolic metamaterial with a response that is a composite of the different layers which are significantly smaller than a wavelength.

The form of the extended NLSE for double negative media is given below ${ }^{28}$ in Eq. (4.1). Note that there is also the capability of adding higher order dispersion and Raman scattering effects in future work

$$
i \frac{\partial \psi}{\partial \mathrm{Z}}-\operatorname{sgn} \frac{1}{2}\left(\beta_{2}\right) \frac{\partial^{2} \psi}{\partial T^{2}}+\operatorname{sgn}\left(\bar{\chi}^{(3)}\right)|\psi|^{2} \psi+v \psi=-i \operatorname{sgn}\left(\bar{\chi}^{(3)}\right)^{2} S \frac{\partial}{\partial \mathrm{T}}\left(|\psi|^{2} \psi\right)
$$

where $\beta_{2}$ is the group velocity dispersion, $\mathrm{S}$ is the self-steepening coefficient which is discussed below. $\bar{\chi}^{(3)}$ is the third order nonlinear Kerr coefficient in which the assumption of a weakly guiding system has been embedded. It should be noted that the self-steepening coefficient $\mathrm{S}$ is ${ }^{28}$ : 


$$
S=\frac{1}{\omega_{0}}\left[2+\frac{\left|v_{p}\right|}{v_{g}}+\left(\frac{\omega}{\mu} \frac{\partial \mu}{\partial \omega}\right)_{\omega_{0}}\right]
$$

where $\omega$ is angular frequency, $\omega_{0}$ is the operational frequency, $v_{p}$ is phase velocity, $v_{g}$ is group velocity and $\mu$ is the permeability and:

$$
\frac{\left|v_{p}\right|}{v_{g}}=-\frac{\beta_{1} \omega_{0}}{\beta_{0}}
$$

where $\beta_{0}$ is the wave number, $\beta_{1}=\left.\frac{\partial k}{\partial \omega}\right|_{\omega_{0}}=\frac{1}{v_{g}}$.

The form of $v$ is ${ }^{28}$ :

$$
v=\frac{v_{\max }}{2}\left(1-\left(\frac{\tanh \left(\mathrm{T}-\mathrm{T}_{v}+\left(\frac{Z}{v_{g}}\right)-2 \Delta T_{v}\right)}{\Delta T_{v}}\right)\right)
$$

The magnetooptic parameters that are set in the simulations are thus: $T_{v}$ the delay of the magnetisation after excitation by the electromagnetic input, $\Delta \mathrm{T}_{v}$ which is the normalised time over which the magnetisation takes place, $\mathrm{v}_{\mathrm{g}}$ the group velocity and $v_{\max }$, the maximum value of magnetisation which is defined $\mathrm{as}^{28}$ :

$$
v_{\max }=\frac{\omega}{c} L_{D} n_{m}^{2} Q_{s a t}
$$

where $L_{D}$ is the dispersion length, $n_{m}$ is the refractive index of the magnetooptic material and $Q$ is the magnetooptic parameter that is usually taken to define the strength of its influence, $Q_{\text {sat }}$ represents the saturation of this parameter.

In order to consider metamaterial effects on the NLSE the Crank-Nicolson method has been implemented to numerically solve the propagation equation. The initial step in the process is hence to numerically solve Eq. 4.1 without any metamaterial additions to the NLSE (see Fig. 4.2(a)). The exact Peregrine soliton ${ }^{29}$ solution, $\psi=\left[1-4(1+2 i z) /\left(1+4 t^{2}+4 z^{2}\right)\right] e^{i z}$, was used as an input condition. This involved selecting the exact solution at a specific distance along the z-axis prior to the peak of the breather and allowing it to (numerically) propagate along $\mathrm{z}$. The results of this are given below using pseudo-colour plots that show the evolution of wave intensity $|\psi|^{2}$ as a function of normalized distance and time. A single peak centred at position $\mathrm{z}=0$ is clearly observed, in agreement with the analytical solution. The next step in the process is to introduce metamaterial effects through the appropriate selection of $\mathrm{S}$ the self-steepening coefficient (which can be negative or positive). In subsequent results magnetooptic effects are added through the values given to $T_{v}$ the delay of the magnetisation after excitation by the electromagnetic input, $\Delta \mathrm{T}_{v}$ the normalised time over which the magnetisation takes place, $v_{\mathrm{g}}$ the group velocity and $v_{\max }$, the maximum value of magnetisation. In Figs.4.2(b) and 4.2(c) it can be seen that the time of arrival of the Peregrine can be changed depending on the values selected for the self-steepening coefficient. It is noted that the slight disturbances of the ideal NLSE propagation induce some deviations from the expected theoretical solution, but when compared to Fig. 1 most of features related to the Peregrine soliton (rogue wave) remain clear. This type of tilted Peregrine soliton structure has also been seen in the case of approximate polynomial rogue-wave solutions obtained beyond the integrable Sasa-Satsuma or Hirota equations ${ }^{29}$. The angle $\theta$ to the $\mathrm{z}$-axis corresponds to an effective velocity proportional to the self-steepening coefficient ${ }^{29} \mathrm{~S}$. 


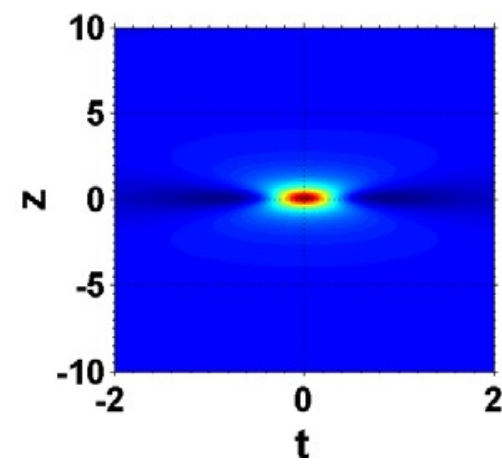

(a)

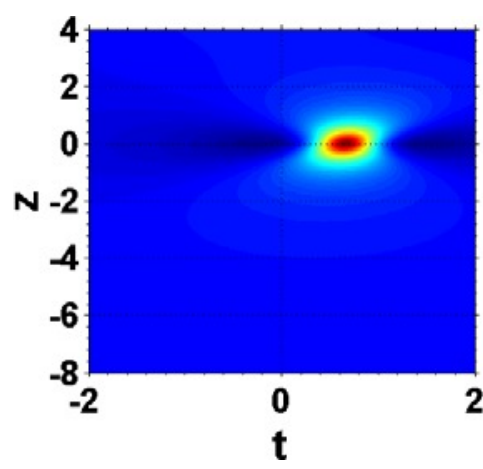

(b)

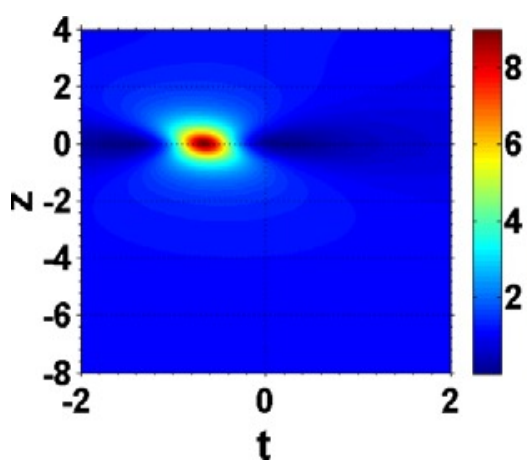

(c)

Fig. 4.2 Numerical simulations showing the effect of self-steepening during propagation (without magnetooptic effect) on the initial excitation of the NLS Peregrine soliton. The exact solution of the Peregrine soliton is chosen as a starting condition at normalized distance $\mathrm{z}=-10$ (other plots have different starting positions but the principle is the same). The Crank-Nicolson technique is then used to solve the extended NSLE (Eq. 4.1) and propagate along the z-direction. (a) Without self-steepening $\mathrm{S}=0$, it can be seen that the result of this process is a single peak at position $\mathrm{z}=0$ that subsequently decays in line with the analytical Peregrine solution. (b) $\mathrm{S}=+0.035$, and (c) $\mathrm{S}=$ -0.035 . Note the opposite time shifting of the intensity peak of near-Peregrine soliton for opposite values of $\mathrm{S}$.

Magnetooptic effects can now be introduced. However, it should be emphasised that, for the magnetooptic effect to operate on the central peak of the Peregrine soliton, a sufficient propagation distance is required, prior to where the peak manifests itself. Figure 4.3(a) shows the effect of using a value of $S=-0.035$ with magnetooptic parameters set as $v_{\max }=-16, T_{v}=200, \Delta T_{v}=10$ and $v_{g}=0.03$. It can be seen that the addition of the magnetooptic parameters moves the peak back to the position $\mathrm{t}=0$, noting that there is an overall clockwise rotation of the rogue wave structure. Figure 4.3b shows the influence of the same magnetic parameters but with the propagation prior to the main peak being 12 dimensionless units along the z-axis rather than 8 . It can thus be seen that significant control can be achieved through metamaterial effects, without cancelling the main features of the rogue wave solution.

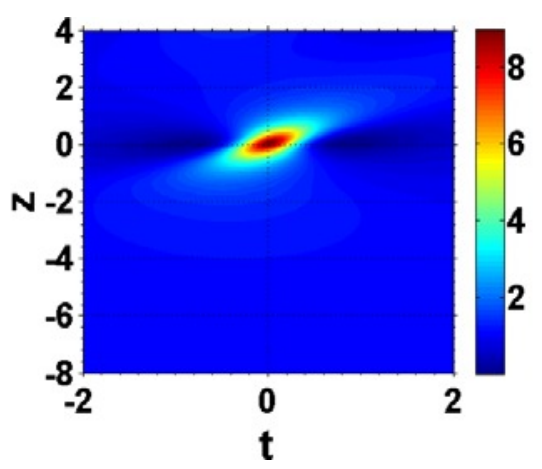

(a)

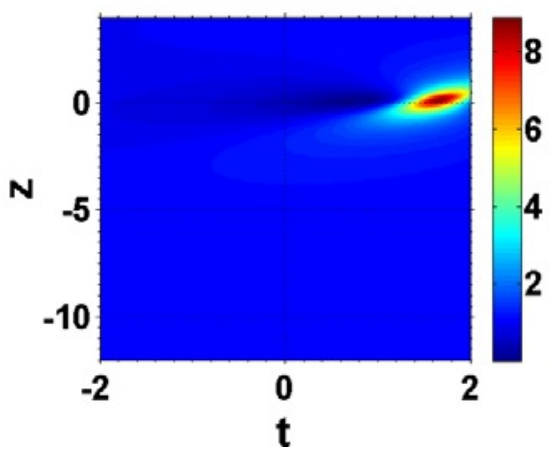

(b)

Fig. 4.3 (a) Effect of magnetooptic parameters set as $v_{\max }=-16, \mathrm{~T}_{v}=200, \Delta \mathrm{T}_{v}=10$ and $\mathrm{v}_{\mathrm{g}}=0.03$ on the self-steepening $\mathrm{S}=-0.035$. The addition of the magnetooptics has moved the peak back towards the origin of the time axis. (b) Same parameters but the difference lies in the distance propagated prior to the peak localization. The magnetooptic effect now moves the peak to the positive part of the time axis.

Other areas of study include the excitation of NLSE breather solutions with non-ideal input conditions ${ }^{29,31,32,33}$ to Eq. (4.1). Our objective is to assess the impact of negative phase nonlinear metamaterials in this area, in particular, in the presence of self-steepening and the addition of magnetooptic parameters. Figure 4(a) shows a simulation 
carried out with the following input condition $^{30}: \psi=\sqrt{1+0.145 \cos (\omega t)}$, where $\omega=2(1-2 a)^{1 / 2}$ and $a=$ 0.25 . This input condition simply corresponds to an intensity-modulated continuous wave with angular frequency $\omega$ linked to the governing parameter $a$ (noting that $a$ determines the physical behaviour of the excited breather solution) and here $a=0.25$. with the excited breather being part of the family of Akhmediev breathers).

Utilising the same process as for the Peregrine solution we show how magnetooptics can be used to cancel the effects of self-steepening for non-ideal starting conditions to the NLSE. First, we observe that non-ideal initial conditions yield periodic evolution as a function of propagation, in contrast to the exact Akhmediev-breather (AB) theory with a single growth/decay cycle of the temporal periodic pattern. However, each growth-return cycle remains well-described by the analytic AB solution. Next, the self-steepening effect is added to the simulation ( $\mathrm{S}$ $=-0.035$ ). Fig. 4.4(b) shows such a scenario where it can be seen that both the first and second set of peaks have been rotated and moved in a negative direction with respect to the time axis. It can be seen that the shift leaves both sets of peaks aligned in the same orientation and rotated counter clockwise. Moreover, the spatial localization of the maximum intensity peak does not change significantly. Now Fig.4.4(c) shows the effect of adding magnetooptic parameters sufficient to move the second set of peaks back to its original position prior to the addition of self-steepening and magnetooptic effect $\left(v_{\max }=-7, \mathrm{~T}_{v}=200, \Delta \mathrm{T}_{v}=10\right.$ and $\left.\mathrm{vg}_{\mathrm{g}}=0.03\right)$. when this has been achieved it can be seen that the first set of peaks has not reached its original position prior to the addition of self-steepening and magnetooptic effects and still has counter clockwise rotation. This again illustrates here that the magnitude of the response from the magnetooptics is position dependent. It also illustrates again that it is possible to almost cancel the effective velocity induced by self-steepening with an appropriate choice of the magnetooptic response.

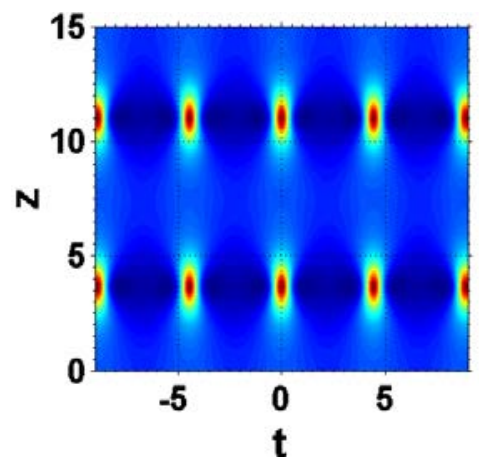

(a)

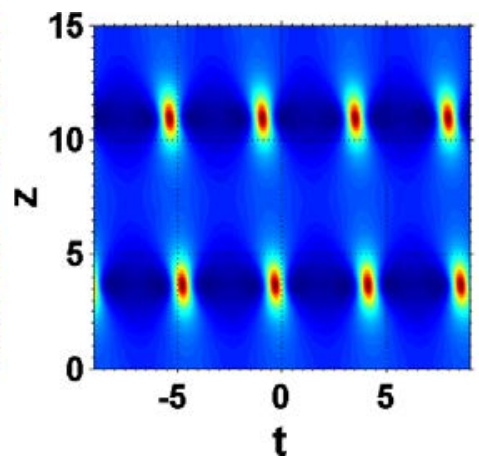

(b)

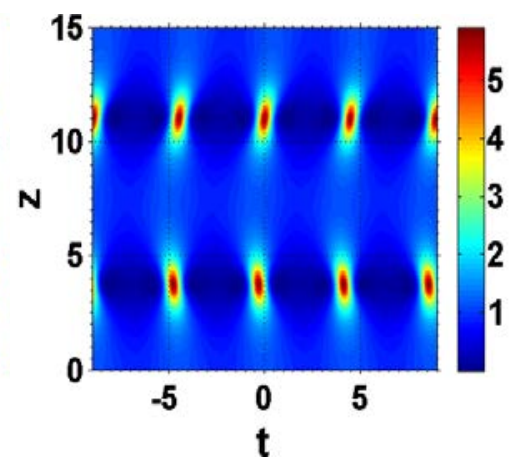

(c)

Fig.4.4. Numerical simulations of Eq. (4.1) with the following input condition $\psi=$ $\sqrt{1+0.145 \cos (\omega t)}$, which corresponds to the non-ideal excitation of an Akhmediev breather $(a$ $=0.25$ ). (a) Without self-steepening and magnetooptic effects, the periodic emergence of nearAkhmediev breathers correlates well with Ref. 30. (b) Impact of self-steepening (with $S=-0.035$ ). It can be seen that both sets of peaks have moved along the time axis in a negative direction. (c) Impact of self-steepening and magnetooptic parameters $\left(\mathrm{S}=-0.035, v_{\max }=-7, \mathrm{~T}_{v}=200, \Delta \mathrm{T}_{v}=10\right.$ and $v_{g}=0.03$ ). There is a clear distinction between how the first and second sets of peaks respond due to the propagation distance along $\mathrm{z}$.

These latter results with the use of non-ideal input conditions thus show similar responses to the exact breather excitation. As has been said, it is thus possible to see how a range of different input conditions could potentially be controlled through the use of magnetooptics. Further the results, in support of previous conclusions ${ }^{6}$, show that the addition of terms to the NLSE describing these phenomena do not prevent the emergence of rogue wave structures almost identical to rogue NLSE breather solutions. The fact that breathers propagating with a certain angle to the line $t=0$ can also be induced by non-ideal input wave excitation, as well as propagation effects, is interesting to note ${ }^{30}$. The resulting inclined trajectory is usually associated with an asymmetric spectrum or/and frequency detuning, with a distinct mean group velocity of the breather under study. Note that rogue wave solutions analysed in the framework of the Sasa-Satsuma equation already revealed strong spectral asymmetries $^{33}$. In future, a detailed analysis of spectral evolution will be mandatory as this information is important for a complete investigation of the rogue wave properties. 
A roadmap, using double negative metamaterials as a guideline, has thus been set out giving the direction for the study of extreme events not only for double negative metamaterials but for hyperbolic media by utilizing similar numerical approaches and tools and then illustrating them by generating some initial results. The numerical propagation tool allows a number of different input conditions to be set at the outset. Thus, it is now possible to look at a variety of different conditions such as the variation of the self-steepening coefficient and also investigate magnetooptic effects. Further, there is now the possibility of studying Raman scattering and quintic nonlinearity. The properties of spatial rogue wave events can also be studied as part of future work. This will be important not only in its own right but also in support of the study and understanding of other types of extreme wave events such as hydrodynamics.

\section{REFERENCES}

[1] Walser, R.M., "Electromagnetic metamaterials,” Proc. SPIE. 4467, 1-15 (2001).

[2] Veselago, V.G., Braginsky, L., Shklover, V., Hafner C., "Negative refractive index materials." Journal of Computational and Theoretical Nanoscience. 3, 189-218 (2006).

[3] Smith D.R., Pendry J.B., Wiltshire M.C.K.,"Metamaterials and negative refractive index," Science 4, 788-92 (2004).

[4] Boardman, A.D., King, N., Velasco L., "Negative refraction in perspective," Electromagnetics 25, 365-89 (2005)

[5] Engheta, N., Ziolkowski R.W., "Metamaterials,” IEEE Press Wiley Interscience (2006)

[6] Boardman, A.D., Alberucci, A., Assanto, G., Grimalsky, V. V, Kibler, B., McNiff, J., Nefedov, I.S., Rapoport, Y.G., and Valagiannopoulos, C.A., " Waves in hyperbolic and double negative metamaterials including rogues and solitons," Nanotechnology 28, 444001 (2017).

[7] Pendry, J. B., "Negative Refraction Makes a Perfect Lens", Physical Review Letters 85, 3966- 3969 (2000).

[8] Veselago, V. G., "The electrodynamics of substances with simultaneously negative values of epsilon and mu", Soviet Physics Uspekhi 10, no. 4 (1968).

[9] Pendry, J. B., "A Chiral Route to Negative Refraction", Science 306, 1353-1355 (2003).

[10] Valagiannopoulos, C. A. and Tretyakov, S., "Emulating hyperbolic-media properties with conventional structures", New Journal of Physics 16, 063004 (2014).

[11] Kidwai, O., Zhukovsky, S. V. and Sipe, J. E., "Dipole radiation near hyperbolic metamaterials: applicability of effective medium approximation", Optics Letters 36, 2530-2532 (2011).

[12] Nefedov, I. S., Valagiannopoulos, C. A. and Melnikov, L.A., "Perfect absorption in graphene multilayers," Journal of Optics 15, 114003 (2013).

[13] Argyropoulos, C., Estakhri ,N. M., Monticone, F., and Alu, A., "Negative refraction, gain and nonlinear effects in hyperbolic metamaterials," Optics Express 21, 15037-15047 (2013).

[14] Jin, B., Guo T and Argyropoulos, C., "Enhanced third-harmonic generation with graphene metasurfaces," Journal of Optics 19, 094005 (2017).

[15] Boyle, J.W. Nikitov, S.A. , Boardman, A.D. , Booth, J.G., and Booth, K.," Nonlinear self - channeling and beam shaping of magnetostatic waves in ferromagnetic films," Phys. Rev. B. 53,12173-12181 (1996).

[16] Grimalsky, V., Rapoport, Yu., and Slavin, A.N.," Nonlinear diffraction of magnetostatic waves in ferrite films," Journ. De Physique IV France. 7, C1-393 - C1-394 (1997). 
[17] Poddubny, A., I. Iorsh, P. Belov and Kivshar, Yu.," Hyperbolic metamaterials, “Nature Photon. 7, 958967 (2013).

[18] Rapoport, Yu., Boardman, A., Grimalsky V., Selivanov ,Yu. and Kalinich, N. ," Metamaterials for space physics and the new method for modeling isotropic and hyperbolic nonlinear concentrators, "Proc. of International Conference on Mathematical Methods in Electromagnetic Theory (MMET), Kharkiv, Ukraine. , 7679 (2012).

[19] Rapoport, Yu.G., Grimalsky, V.V. Koshevaya, S.V., Boardman, A.D. and Malnev, V.N.," New method for modeling nonlinear hyperbolic concentrators," Proc. of IEEE 34th International Scientific Conference on Electronics and Nanotechnology (ELNANO). Kyiv, Ukraine, 35-38 (2014).

[20] Rapoport, Yu. G., Boardman, A.D., Grimalsky, V.V., Ivchenko, V.M. and Kalinich, N.,”Strong nonlinear focusing of light in nonlinearly controlled electromagnetic active metamaterial field concentrators," J. of Opt. (United Kingdom) 16, 0552029-0552038 (2014).

[21] Caloz, C., and Itoh, T., “ Electromagnetic Metamaterials: Transmission Line Theory and Microwave Applications (New Jersey: Wiley-IEEE Press), ”376 (2006).

[22] Marchuk, G. I., Edited by Ciarlet, P. G. and Lions, J. L.," Splitting and Alternating Direction Methods, Handbook of Numerical Analysis ," 1, 197-462 (1990).

[23] Rapoport Y.G., Grimalsky V.V., Boardman A.D., and MalnevV. N., (2014). Controlling nonlinear wave structures in layered metamaterial, gyrotropic and active media, Proc. of IEEE 34rd International Scientific Conference Electronics and Nanotechnology, ELNANO, April 15-18, 2014, Kyiv, Ukraine, P. 46-50.

[24] Yang X, Yao J, Rho J, Yin X and Zhang X, (2012) Experimental realization of three-dimensional indefinite cavities at the nanoscale with anomalous scaling laws. Nat. Photon. $6450-4$

[25] Yao J, Yang X, Yin X, Bartal G and Zhang X, (2011) Three dimensional nanometer-scale optical cavities of indefinite Medium. Proc. Natl Acad. Sci. 108 11327-31

[26] Karaaslan M and Bakir M, (2014). Progress In Electromagnetics Research. 149 55-67

[27] Melik R Unal E, Perkgoz N K Puttlitz C and Demir H V, (2009). Metamaterial-based wireless strain sensors. Applied Physic Letters 95, $011106 \_2009$

[28] Boardman AD, Hess O, Mitchell-Thomas RC, Rapoport YG, and Velasco L, (2010). Temporal Solitons in Magnetooptic and metamaterials waveguides, Photonics and Nanostructures - Fundamentals and Applications 8, P. 228-43.

[29]Kibler B, Fatome J, Finot C, Millot G, Dias F, Genty G, Akhmediev N, and Dudley JM (2010). The Peregrine soliton in nonlinear fibre optics. Nat. Phys. 6, P. 790-5

[30] Ankiewicz A, Soto-Crespo JM, Chowdhury MA, and Akhmediev N, (2013). Rogue waves in optical fibers in presence of third-order dispersion, self-steepening, and self-frequency shift. J. Opt. Soc. Am. B 30, P. 87-94

[31] Kibler B, Fatome J, Finot C, and Millot G, (2016). Rogue and Shock Waves (Ed., Onorato M), Lectures Notes in Physics, Springer, 926, P. 89.

[32] Akhmediev N, Kibler B, Baronio F, et al, (2016). Roadmap on Optical Rogue Waves and Extreme Events. J. Opt. 18, P. 063001.

[33] Akhmediev N, Soto-Crespo JM, Devine N, Hoffmann NP, (2015). Rogue wave spectra of the Sasa-Satsuma equation. Phys. D 294, P. 37-42. 